\title{
ALASDAIR MACINTYRE: PERSONA E PERSONALISMO
}

\author{
Alasdair Maclntyre: person and personalism
}

Rocco Carsillo

Università di Chieti (Itália)

\begin{abstract}
Somario: Maclntyre è il grande avversario lottatore contro modernità e post-modernità. Egli scopre, ed argomenta, che all'origine della affermazione "moderna" c'è una grande truffa fondata sulla presunzione di liberarsi dalla soggezione alla storia, intesa sia come Tradizione sia come metodo con una sua epistemologia per comprendere l'uomo e il suo operare. L'epilogo di tale pretesa è sotto gli occhi: un razionalismo autoreferenziale che non ha più contatto con la realtà e che proprio per questo si riduce a delirio di onnipotenza o di potenza decaduta per resistenza indebita di storia e tradizioni "portatrici solo di oscurantismo!". La realtà porta, invece, a scoprire l'identità dell'uomo come «io» contestualizzato, che nasce da una storia, una storia di amore, e si realizza in un contesto di condizioni e condizionamenti, con cui bisogna sempre fare i conti se si desidera essere lealmente attenti a cogliere le notizie che da essa provengono per comprendere chi è questo essere speciale e particolare. Anche altri filosofi hanno risposto alla "pretesa modernista", però spesso o con un atteggiamento di "falso irenismo", teorizzando un dialogo con cui, prendendo a prestito il metodo illuministico, si è cercato di asserirne "dialetticamente" la falsità, cercando di trattenerne, invece, "le cose buone"; oppure ponendosi come pensatori alternativi al pensiero "comunista" distruttore della "comunità» e della "persona». Secondo Maclntyre, l'errore di questi è nel non aver inteso che la falsità "moderna" è proprio nel suo metodo che ha posto come a-priori non negoziabile, in ogni riflessione e ricerca, il rifiuto della storia, della concretezza, del particolare, della tradizione, propugnando che solo la "spersonalizzazione", la "universalizzazione", insomma: solo il regno della "ragion pura" e autopurificatasi ha ragione e dignità di essere l'a-priori per eccellenza nella ricerca filosofica. II "personalismo", nella versione europea, non raramente è incorso in questa confusione.
\end{abstract}

Parole chiave: MacIntyre, Persona, Personalismo.

Abstract: Maclntyre is the big fighter against modernity and post-modernity. He discovers, and argues that in the origin of the "modern" statement there's a big scam founded on the presumption of freeing from subjection to history, understood both as a tradition and as a method with his epistemology to understand the man and his agency. The epilogue of this claim is plain: a self-referential rationalism that has no more contact with reality, and for this reason comes down to illusion of omnipotency or fallen power for improper resistance of history and traditions "only bearers of obscurantism!." The reality leads, however, to discover the identity of the man as an "l" in context, which comes from a story, a story of love, and which is realized in the context of conditions and constraints; which must always be 
reckon with if you want to be fairly careful to seize the news that it has come from it to understand who is this special and unique being. Other philosophers also answered to that "claim modernist", but often or with an attitude of "false irenicism", by theorizing a dialogue which, borrowing the Enlightenment method, attempted to affirm "dialectically" that falsehood, by keeping however its "good things"; or acting as alternative thinkers to the "communist" thought destroyer of "community" and "person". According to Maclntyre, their error is not having understood that the "modern" falsehood is right in its approach itself, which has placed as a-priori not negotiable in any reflection and research the refusal of history, the concrete, the particular, the tradition, by advocating only the "depersonalization", the "universalization", in short, only the realm of "pure reason" and self-purification has the right and dignity of being the a-priori for excellence in philosophical inquiry. The "personalism" in the European version not rarely ran into this misunderstanding.

Keywords: MacIntyre, Person, Personalism

\section{Storia "personale" nella Tradizione.}

È in Scozia che Maclntyre "incontra" e conosce le «tradizioni» che lo hanno generato e al cui studio si è dedicato con passione, e che sono state per lui punto di riferimento per ogni ulteriore sviluppo e consolidamento della sua ricerca filosofica per giungere alla "Tradizione". È la mentalità, la lealtà, la spontaneità, la fedeltà degli uomini e delle donne che vivono all'interno di quelle storie che lo commuovono e gli mettono nel cuore e nella mente quello stupore che, a sua volta, è naturale in chi riconosce in "quella novità" quello che aveva sempre cercato. In tal modo, questo fatto diventa il punto fermo di partenza per la sua ricerca; anzi lo scoprirà come il paradigma di ogni seria ricerca: perché, secondo Maclntyre, l'epistemologia non può nascere ed esaurirsi dalla e nella correttezza logica e corrispondenza autoreferenziale con i principi, ma dallo stimolo che viene dalla storia ad indagare e scoprire, e dalla corrispondenza di questa con quello che «è, fu e sarà» il "cuore" vero dell'essere umano.

L'educazione di Maclntyre è ispirata al modello delle tradizioni celtiche di cui sente profondamente il fascino e l'appartenenza, e delle quali, proprio per questo, vuol scoprire radici e consistenza.

Lo stupisce la ricchezza morale nella vita e nella storia condivisa di queste comunità perché in esse accade, in maniera tranquilla e costruttiva, il perseguimento 
personale e comune di beni umani in un contesto di collaborazione reciproca e di condivisione della concezione del «bene», vi fioriscono le virtù considerate sia come frutto dello crescita personale di ognuno ma anche come condivisi modelli di eccellenza umana da imitare, insomma, dei percorsi concreti che plasmano la vita e l'educazione e che, in quanto tali, costituiscono il bene significativo delle stesse comunità partecipato da ognuno dei membri.

È inizialmente cristiano secondo il «credo» presbiteriano, poi diventa anche marxista, ma nessuna delle due «proposte», ognuna per i propri limiti contraddittori, è capace di accompagnarlo in maniera esauriente nella ricerca filosofica, e, pertanto, nessuna gli andrà bene: l'approdo alla Fede Cattolica, invece, è un punto di arrivo che corrisponde alle sue attese speculative e teoretiche fondamentali.

È «uno spirito moderno», ma solo nel senso che, pur essendo un grande entusiasta della storia e della vitalità della Tradizione, non si rassegna ad esserne un mero laudator: egli la contempla nel suo nascere e svilupparsi, ma ne analizza la capacità epistemologica per la comprensione dell'uomo e del suo agire morale nel loro essere e nei loro contenuti; al punto che la cifra per comprendere la tipicità della sua ricerca filosofica è il ricupero della dignità razionale della «tradizione», della capacità veritativa della "storia" concreta delle persone, inintelligibili senza l'aggancio con le origini e con il contesto, e questo come argomento forte per superare l'intellettualismo razionalistico e riduttivo dell'universalismo kantiano, ma anche per sfuggire alle maglie insidiose della riduzione relativistica.

La riflessione sulla "tradizione" e il metodo usato, non hanno nulla a che vedere con il metodo illuministico: non la considera come modello arcaico di vita legato ad un tempo passato, ma come «luogo» dell'essere e del vivere, come «luogo» del divenire e del configurarsi dell'uomo e della persona; come «luogo» in cui vengono elaborate e scoperte le ragioni valide e garantite della vita; per cui, è la conclusione di MacIntyre, la loro razionalità è intuibile, ma solo a posteriori, per la capacità di giustificarsi come "epistemologia" della vita. Ed è con questo animus che ricerca e 
approfondisce tutte le tradizioni, non solo quelle classiche, ma anche quelle recenti e recentissime, sì che il giudizio che ne deriva è storico, ma, soprattutto, anche teoretico e «completo».

All'inizio degli anni Settanta del secolo XX Maclntyre si trasferisce negli Stati Uniti dove matura definitivamente il suo pensiero: l'approfondimento delle teorie di Kuhn sulla storia dei "paradigmi scientifici" costituisce una scoperta interessante il cui contenuto verrà usato per approfondire la sua posizione originale contro il relativismo teoretico ed etico.

In questo contesto non ha paura di cambiare strada quando su un'altra scopre una luminosità più intensa della verità che cerca: questo cambiamento, anzi, può essere visto come segno di lealtà verso quella stessa verità, per la fedeltà alla quale non si risparmia di perdere anche la faccia.

\section{Persona, Personalismo ed Etica "moderna"}

II «personalismo", così come storicamente lo conosciamo, come concezione e come "teoria esplicativa», non fa parte del background di Maclntyre. La concezione metafisica di Mounier è solo parzialmente condivisa da lui, e solo per quello che, riferendosi al pensiero di Aristotele, costituisce il fondo comune condiviso da tutti i "metafisici". La Metafisica che è al fondo della riflessione macintyriana è di stampo storicista, assolutamente inedita come metodo ed ampia come campo di ricerca, che raggiunge la sua "sistemazione" solo «a posteriori». Secondo Maclntyre, essa si impianta solo a seguito di una ricerca seria e teoretica sulla realtà storica, in cui si cerca di evincerne i connotati indistruttibili attraverso il metodo aristotelico della epagoge e della dialettica, come tentativo di ordinare le singole tappe in vista del telos secondo il contesto teoretico vissuto all'interno di un ethos. Non è quindi una riflessione sulla realtà a partire da principi posseduti a priori, ma una riflessione indotta dalla realtà, la quale è essa stessa che, contemplata ed accolta con libertà e lealtà, cioè, senza l'imbroglio dell'ideologia e della falsa coscienza, si incarica di svelarci tutta se stessa. 
Nella modernità, invece, la "teoria» è il punto di partenza organizzato e costruito dagli intellettuali o dagli scienziati entro cui collocare in sequenza presunta causale e razionale i dati raccolti dall'esperienza, i quali serviranno o per confermarla o per falsificarla, sicchè la storia potrebbe essere, da questo punto di vista, un museo di teorie, più o meno valide; inoltre, non raramente, nella modernità la teoria potrebbe essere anche il punto di arrivo escogitato, pure esso a priori, per offrire una spiegazione razionale dei dati, molto spesso solo razionalizzati ad uso ideologico o utopico. E in Mounier questo tipo di "contaminazione modernista" è molto presente, anche se la sua concezione è, per tanti versi, affascinante.

Pertanto, Maclntyre non sposa la causa «personalistica», ma

1) sia la sua originale concezione del "soggetto morale", della "morale", della "comunità" e della "tradizione",

2) così come la sua critica spietata verso il razionalismo tendente inesorabilmente al nichilismo "liberale" o nietzschiano, ${ }^{1}$ con la conseguente distruzione della possibilità di capire il soggetto nella sua storicità concreta,

3) sia la sua critica verso il riduzionismo empiristico, positivistico e neo-positivistico, tendenti verso la chiusura individualistica e «scientifica» di ognuno "nel suo particulare", eliminando ogni possibilità che l'uomo in quanto tale possa riferirsi alla trascendenza, lo mettono inevitabilmente in rapporto teoretico con lo stesso "personalismo" su questioni non marginali.

Maclntyre vede "l'etica moderna" come un discorso di presunta "emancipazione" che, in seguito e a causa delle varie teorie illuministiche, si è preoccupata di "liberare" la filosofia dalla superstizione metafisica della "Tradizione", per la quale, invece, è fondamentale considerare l'uomo sia come è di fatto, cioè nella sua realtà storica, sia come deve essere, cioè nella sua realtà di "progetto", sia cosa deve fare per realizzare il suo dover essere, cioè come programma di vita; ${ }^{2}$ ma "l'etica moderna" ha pure declassato la dimensione teleologica da proprium della natura

\footnotetext{
${ }^{1}$ Vedere a riguardo tutto il capitolo IX di Dopo la virtù.
}

${ }^{2}$ Dopo la Virtù, p. 71. 
umana in quanto tale a pura "emozione" intima e privata, ed ha inteso considerare l'azione dell'uomo, o

1) come mezzo per soddisfare bisogni, desideri e piaceri, in una teorizzazione utilitaristica, 0

2) come concretizzazione della propria moralità, in una visione deontologistica, o

3) come espressione della integrazione sociale, in una concezione contrattualistica.

Per tutto questo, l'antropologia "moderna" ha di fatto ridotto la riflessione sull'uomo ad una mera presa d'atto della sua presenza fattuale, con la conseguenza che la vita umana, soprattutto sociale, viene ormai regolata solo da un rigido e voluminoso apparato normativo che varia a seconda delle antropologie in campo: in fondo, il "fatto uomo" è solo descritto; per la comprensione "di lui" le uniche abilitate a farlo sono le "scienze descrittive", con al massimo appendici meccanicistiche con apparente richiamo ad una causalità "superiore", ma solo per spiegarne i fenomeni, e nulla più. La morale è ridotta, nella migliore delle ipotesi, ad essere un compendio di regole da apprendere ed applicare per "educare" I'uomo e migliorarlo. ${ }^{3}$

Bisogna ancora aggiungere che questa morale è risultata ingannevole perché, di fatto, ha finito con l'esaltare solo la "volontà di potenza", dei singoli o delle collettività, la quale volontà, nelle sue scelte, non deve render conto ad altri che a se stessa, dal momento che la persona è stata trasformata in in-dividuo, solo e indiviso con se stesso, ma diviso e separato da ogni altra cosa, proprio per il fatto che ha inteso "liberare" la persona

- da ogni legame con l'intelligenza metafisica, temendo che questa potesse giudicare erroneo il contenuto delle sue scelte legate invece solo al desiderio;

- dalla Tradizione, ritenuta oscurantista e incapace di rendere l'uomo veramente libero nell'oggi;

- dalla polis, considerando questa solo per i rapporti "secondari" tra i singoli membri guidati da "interessi", temendo che il considerarla secondo la concezione

\footnotetext{
${ }^{3}$ Enciclopedia, Genealogia e Tradizione, p. 198.
} 
tradizionale gli potrebbe impedire, in nome del bene comune, di godere di "ogni" bene.

Questa concezione, che si collega direttamente a Kant, è ritenuta come un inno alla libertà, ma a quale libertà?

In fondo, è la critica di Maclntyre, la modernità, dopo averlo anemizzato, pone l'individuo in solitudine "ontologica" ed ermeneutica di fronte alle regole, o, nella migliore delle ipotesi, di fronte al giudizio categorico della sua ragione, ed in questa solitudine, nonostante l'indisponibilità a rinunciare alla libertà individuale, visto, però, che ognuno la possiede uguale, onde salvaguardare almeno la propria sopravvivenza, è disposto hobbesianamente ad autolimitarsi con l'adozione di regole comuni, il cui potere, anche coercitivo, mira solo a rendere possibile la tranquilla convivenza degli individui, soli e ugualmente liberi. La conseguenza? È stato annullato ogni motivo condiviso per agire guidati da tensioni ultraindividuali, al di là di quelli puramente formali e impersonali dell'apparato normativo. L'etica moderna si è, cioè, ridotta ad un discorso minimale di rispetto degli altri, possibilmente, direbbe Kant, visti come soggetti e non come strumenti. Ma questo cambiamento, prodotto nella società dalla conseguente riduzione dell'etica a deontologia, ${ }^{4}$ che prevede l'agire dell'uomo come esecuzione di doveri, porta anche all'im-personalizzazione delle decisioni e delle azioni, porta allo scardinamento radicale della stessa azione da ogni riferimento a ragioni morali; e la sua legalità, essendo un problema solo di coerenza con delle regole e doveri corrispondenti ma irriferibili a ragioni superiori, sarà ugualmente valida, lecita, legittima e garantita. Infine, questa teorizzata im-personalizzazione, occorre aggiungere, è qualcosa di ancora più profondamente dissolutore della osservanza solo esteriore, in cui si pretende un giudizio di valore e di merito formalmente "distaccato" sulle azioni compiute; nella prospettiva im-personale, infatti, può essere evocata, al massimo, una tutela solo giuridica delle regole comuni in vista del retto funzionamento delle relazioni interpersonali previste dalle regole stesse, ma l'individuo viene

\footnotetext{
${ }^{4}$ Cfr. a riguardo il mio libro Deontologia tra Etica e Diritto, pubblicato in Italia nel 2011.
} 
sollecitato ad auto-convincersi che così è e così deve essere: si moltiplicheranno le "etiche" e le "bio-etiche", ma saranno solo regolamenti convenzionali da applicare nelle varie situazioni per garantire nelle stesse uguale fruizione dei beni previsti, fondate come modi partecipati e "corretti" giustificati dal richiamo a principi universali la cui interpretazione, prevista per definizione "pluralista", elimina proprio la loro funzione di principi. La persona sarà soggetto morale, ma di una moralità che si esprimerà come tale solo per l'osservanza del dovere "colto" attraverso il rapporto immediato con l'universalità della legge.

Ora, questo individualismo, corrispondente al modello della "universalità impersonale", comporta che «l'in-dividuo», sia l'unica certezza dal lato del soggetto, mentre dal lato della socialità, per garantirne la libertà e l'autonomia, ma con misura, viene teorizzata "l'impersonalità delle norme" che ne regolano i comportamenti. Ma, poiché tra l'in-dividuo e la società, al di là dell'occasionalità degli interessi, non c'è alcun ordine oggettivo capace di motivare, guidare e regolare le relazioni intersoggettive, queste vengono previste fondate solo sulle "libere" decisioni del soggetto, il quale, liberato dalla pregnanza metafisica, e non avendo più consistenza ontologica, ma solo "noumenica", mentre teoricamente viene riconosciuto come volontà libera ed autonoma che sceglie solo in nome di se stesso, e quindi senza "ragioni esterne", ciò da cui ultimamente ed effettivamente è orientato come fattore decisivo e decisionale è una reale "irrazionalità": preferenze emotive, opinioni ed interessi, preoccupazione di soddisfare desideri e bisogni. Quali? Quelli che nell'attimo appaiono i più intelligenti, impellenti e decisivi.

Questo «io» che decide ed agisce così, da MacIntyre è definito «io emotivista»: chiuso e assoluto nella sua immediatezza, senza mediazioni con il mondo esterno e con gli altri, un «io narcisista». 


\section{Pensiero di Maclntyre.}

In contrasto con questa visione, Maclntyre propone la sua versione forte dell'etica che si radica in Aristotele e S. Tommaso.

Come individuare e configurare il "soggetto morale"? Maclntyre risponde con una riflessione che spazia su diversi livelli.

Innanzitutto: la questione del soggetto è una questione ontologica, mentre, invece, l'io specificamente moderno si impone "decostruito" e diverso rispetto all'immagine pre-moderna dell'io. ${ }^{5}$ Poi, c'è l'aspetto morale per cui il soggetto entra in relazione con il piano dei beni ( $\rightarrow$ dei valori) in vista del proprio bene da perseguire e raggiungere, e questo sia in rapporto al proprio giudizio morale sia rispetto ai giudizi morali della tradizione di appartenenza.

Altro approccio caratteristico è la convinzione che la dimensione morale non è un fattore astratto o considerabile solo in sé per una sua intelligibilità universale, e quindi attingibile da ognuno che lo voglia e lo possa, ma è piantata e incarnata in una comunità da cui, solo, la persona l'attinge ed apprende. La dimensione morale ha, cioè, una originale dimensione storica e sociale, poiché, dice Maclntyre, non esiste e non si può configurare un giudizio morale al di fuori di una concreta tradizione morale. Mentre invece l'etica moderna, "etica delle regole", visione individualistica dell'agire per cui il soggetto decide in modo autonomo confrontandosi in solitudine con un codice di norme e di doveri, ha come suo problema vitale quello di stabilire i limiti di quella libertà che ha teorizzato come assolutamente autonoma; e pertanto a cosa potrà richiamarsi? In nome di che potrà farlo? A quale tipo di convenienza dovrà legarsi? Non è difficile evincere da ciò che molto spesso la libertà dell'uomo è per i moderni moralisti un problema e non una risorsa sociale!

Macintyre propone, invece, di ricuperare la visione del soggetto morale come persona, tipica di Aristotele: il soggetto ricuperato nella sua identità unitaria, nella sua

${ }^{5}$ Dopo la virtù, p. 50. 
unità storica e psicologica, nella sua dignità ontologica, nella sua tensione innata verso il vero e il bene, nella sua capacità di scelta responsabile verso "un" bene conosciuto, riconosciuto come degno "del bene", e perseguito come tale; un soggetto che, pur avendo una coscienza contestualizzata e socialmente caratterizzata, non può non essere riconosciuto pienamente responsabile di sé e del suo agire.

Occorre rimettere al centro di ogni considerazione la persona con tutte le sue dimensioni: la capacità di amare, apprezzare, desiderare ciò che è bene e giusto, la capacità di comprendere la verità e il bene, la capacità di decidere, per cui si riesce a cogliere che le in-tenzioni dell'agente, che fanno di lui un soggetto aperto alla totalità, non sono rivolte immediatamente all'osservanza del dovere, ma alla realizzazione di una vita bella, piena e degna delle sue capacità ed attese personali.

\section{Riferimenti bibliografici}

CARSILLO, R. I/ problema morale in Maclntyre, Levante, Bari, 2000. Filosofia morale, educazione e politica "moderne". Con Maclntyre: oltre il Comunitarismo e il Liberalismo, Levante, Bari, 2007. . Deontologia: tra Etica e Diritto, Aracne, Roma, 2011.

MacINTYRE, A. After Virtue. A Study in Moral Theory, Duckworth, London, 1981,1985²; tr. it. di P. Capriolo, Dopo la virtù. Saggio di teoria morale, Feltrinelli, Milano, 1987. Whose Justice, Which Rationality?, Notre Dame University Press, Notre Dame, 1988; tr. it. di C. Calabi, Giustizia e Razionalità, 2 voll., Anabasi, Milano, 1995. . Three Rival Versions of Moral Enquiry (Encyclopaedia, Genealogy and Tradition), Duckworth, London, 1990; tr. it. di M. D'Avenia, Enciclopedia, Genealogia e Tradizione. Tre versioni rivali di ricerca morale, Massimo, Milano, 1993. 